\title{
Resolved Imaging of the AR Puppis Circumbinary Disk*
}

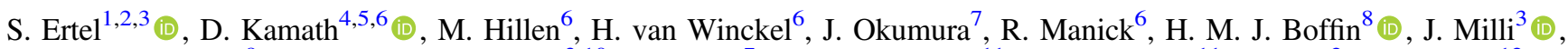 \\ G. H.-M. Bertrang 9 (10), L. Guzman-Ramirez ${ }^{3,10}$, J. Horner ${ }^{7}$ (1) J. P. Marshall ${ }^{11}$ (1) P. Scicluna $^{11}$, A. Vaz ${ }^{2}$, E. Villaver ${ }^{12}$ (1), \\ R. Wesson ${ }^{3,13}$, and S. Xu ${ }^{8,14}$ (iD) \\ ${ }^{1}$ Large Binocular Telescope Observatory, 933 North Cherry Avenue, Tucson, AZ 85721, USA \\ ${ }^{2}$ Steward Observatory, Department of Astronomy, University of Arizona, 993 N. Cherry Avenue, Tucson, AZ 85721, USA; sertel@email.arizona.edu \\ ${ }^{3}$ European Southern Observatory, Alonso de Córdova 3107, Vitacura, Casilla 19001, Santiago 19, Chile \\ ${ }^{4}$ Research School of Astronomy and Astrophysics, Australian National University, Cotter Road, Weston Creek ACT 2611, Australia \\ 5 Department of Physics and Astronomy, Macquarie University, Sydney, NSW 2109, Australia \\ ${ }^{6}$ Instituut voor Sterrenkunde, K.U.Leuven, Celestijnenlaan 200D bus 2401, B-3001 Leuven, Belgium \\ ${ }^{7}$ Centre for Astrophysics, University of Southern Queensland, West Street, Toowoomba, QLD 4350, Australia \\ ${ }^{8}$ European Southern Observatory, Karl-Schwarzschild-Strasse 2, D-85748 Garching bei München, Germany \\ ${ }_{9}^{9}$ Max Planck Institute for Astronomy, Königstuhl 17, D-69117 Heidelberg, Germany \\ ${ }^{10}$ Leiden Observatory, Leiden University, Niels Bohrweg 2, 2333 CA Leiden, The Netherlands \\ ${ }^{11}$ Academia Sinica Institute of Astronomy and Astrophysics, 11F of AS/NTU Astronomy-Mathematics Building, No.1, Section 4, Roosevelt Road, Taipei 10617, \\ Taiwan, ROC \\ 12 Universidad Autónoma de Madrid, Departamento de Física Teórica, E-28049 Madrid, Spain \\ ${ }^{13}$ Department of Physics and Astronomy, University College London, Gower Street, London WC1E 6BT, UK \\ ${ }^{14}$ Gemini Observatory, 670 N. A'ohoku Place, Hilo, HI 96720, USA \\ Received 2018 November 7; revised 2019 January 11; accepted 2019 January 12; published 2019 February 12
}

\begin{abstract}
Circumbinary disks are common around post-asymptotic giant branch (post-AGB) stars with a stellar companion on orbital timescales of a few 100 to few 1000 days. The presence of a disk is usually inferred from the system's spectral energy distribution and confirmed, for a sub-sample, by interferometric observations. We used the SpectroPolarimetric High-contrast Exoplanet REsearch (SPHERE) instrument on the Very Large Telescope to obtain extreme adaptive optics assisted scattered light images of the post-AGB binary system AR Puppis. Data have been obtained in the $V, I$, and $H$ bands. Our observations have produced the first resolved images of AR Puppis's circumbinary disk and confirm its edge-on orientation. In our high-angular-resolution and high-dynamic-range images we identify several structural components such as a dark mid-plane, the disk surface, and arc-like features. We discuss the nature of these components and use complementary photometric monitoring to relate them to the orbital phase of the binary system. Because the star is completely obscured by the disk at visible wavelengths, we conclude that the long-term photometric variability of the system must be caused by variable scattering, not extinction, of starlight by the disk over the binary orbit. Finally, we discuss how the short disk lifetimes and fast evolution of the host stars compared to the ages at which protoplanetary disks are typically observed make systems like AR Puppis valuable extreme laboratories to study circumstellar disk evolution and constrain the timescale of dust grain growth during the planet formation process.
\end{abstract}

Key words: binaries: close - circumstellar matter - planetary systems - stars: AGB and post-AGB - stars: individual (AR, Pup)

\section{Introduction}

Low- and intermediate-mass stars (around 1-8 $M_{\odot}$ ) have their nuclear-burning lives terminated by stellar wind mass loss on the Asymptotic Giant Branch (AGB) if they are single stars, or possibly by mass transfer to a companion if they are binary stars. When the hydrogen-rich envelope of the AGB star becomes very small due to mass loss, the star can no longer maintain its large radius and it shrinks. The shrinking causes an increase in $T_{\text {eff }}$ as the object evolves through the postAsymptotic Giant Branch (post-AGB) phase, up to $T_{\text {eff }} \sim 3$ $10^{4} \mathrm{~K}$ (see van Winckel 2003 for a review), followed by the planetary nebula and white dwarf phases. Binarity is thought to be the main driver for breaking the initially largely spherical envelopes of AGB stars to form bipolar protoplanetary and planetary nebulae (Soker 2004, 2006; Nordhaus \& Blackman 2006; De Marco 2009; Boffin et al. 2012). Some binary stars

Based on observations made with ESO Telescopes at the La Silla Paranal Observatory under program IDs 097.D-0385 (SPHERE) and 094.D-0865 (PIONIER). will already interact when the primary expands during its evolution on the Red Giant Branch (RGB). Such systems, the dusty post-RGB stars, have recently been identified in the Magellanic Clouds (Kamath et al. 2016; Manick et al. 2018). However, due to observational limitations, the binary nature and orbital parameters of these systems are yet to be fully explored.

Many close post-AGB binaries display disk-type spectral energy distributions (SEDs). These are characterized by a clear near-infrared excess, indicating that a fraction of the expelled circumstellar dust and gas must be located close to the central star, near the sublimation radius (van Winckel 2003). It is now well established that this feature in the SED indicates the presence of a stable, compact, Keplerian circumbinary disk, and these sources are referred to as disk sources (e.g., de Ruyter et al. 2006; Hillen et al. 2014; Kamath et al. 2014, 2015). All non-pulsating Galactic post-AGB disk sources are proven to be binaries with binary orbital timescales of the order of one to several years, and therefore this specific characteristic of the SED is closely related to the binary nature of the central star (van Winckel et al. 2009; Gezer et al. 2015). Many pulsating 
objects were also proven to be binaries (e.g., Gorlova et al. 2014; Manick et al. 2017); however, this is more difficult to prove, as large amplitude pulsations (e.g., of RV Tauri stars) induce large radial velocity variations. Another interesting property common to most post-AGB objects with disk-type SEDs is a peculiar photospheric composition depleted of refractory elements (Gezer et al. 2015; Oomen et al. 2018, Kamath \& Van Winckel 2019, and references therein).

Initially, the study of the morphology and evolution of postAGB binaries and their disks was limited to the analysis of spatially unresolved data of the star and infrared excess from the circumstellar dust. From this, disk sizes (outer radii) of a few 100-1000 au were inferred (de Ruyter et al. 2006). CO rotational mapping has confirmed the Keplerian rotation in a sample of systems (Bujarrabal et al. 2013a) and succeeded to resolve the large scale gas disks and their kinematics in several systems (Bujarrabal et al. 2003, 2013b, 2015, 2016, 2017, 2018). Only one similar disk around the central star of the Red Rectangle (HD 44179) could be imaged in scattered light with the Hubble Space Telescope (HST) (Osterbart et al. 1997; Cohen et al. 2004). The first spatially resolved size measurements of the inner dust disks in other systems were obtained using optical long baseline interferometry at infrared wavelengths (Deroo et al. 2006). The same technique provided a more detailed view of selected disks (Deroo et al. 2007; Hillen et al. 2014, 2015) and basic size measurements of the disks in 19 systems (Hillen et al. 2017). The inner rim of the disk in the IRAS 08544-4431 post-AGB system, as well as its inner binary, were resolved in the $H$ band using interferometric imaging with the Very Large Telescope Interferometer (VLTI) /Precision Integrated Optics Near-Infrared ExpeRiment (PIONIER) (Hillen et al. 2016). However, interferometric data are unable to provide the high-dynamic range and image fidelity that direct imaging can provide.

Disks around two less evolved giant stars have recently been imaged using VLT/Spectro-Polarimetric High-contrast Exoplanet REsearch (SPHERE): $\mathrm{L}_{2}$ Pup is an AGB star (Kervella et al. 2015), and BP Psc is a first ascent giant star (Zuckerman et al. 2008; de Boer et al. 2017; Gaia Collaboration 2018). We discuss these cases and compare them to the post-AGB binary disks in Section 5.1.

In this paper, we present the first spatially resolved images of the disk around the post-AGB star AR Pup. The SED of this system suggested a disk orientation close to edge-on (Hillen et al. 2017), which is a favorable case for direct imaging because the disk blocks the direct starlight. We observed the star for our pilot program to demonstrate the feasibility of resolved imaging of post-AGB binary disks in the visible using extreme adaptive optics (AO) systems on $8 \mathrm{~m}$-class telescopes. In Section 2 we present a brief summary of the current literature on AR Pup, relevant to this study. We discuss our observing strategy and data reduction in Section 3. The resulting images are presented and the observed structural components of the disk are discussed in Section 4. In Section 5 we discuss the connection of our imaging results with other observables of the system, specifically the shape of its SED and the photometric variability. In Section 5.3 we highlight the prospects of using post-AGB binary disks as a laboratory to studying circumstellar disk evolution-in particular dust grain growth - and discuss them in the context of potential scenarios of second-generation planet formation on the stellar post-main sequence. We present our conclusions in Section 6.

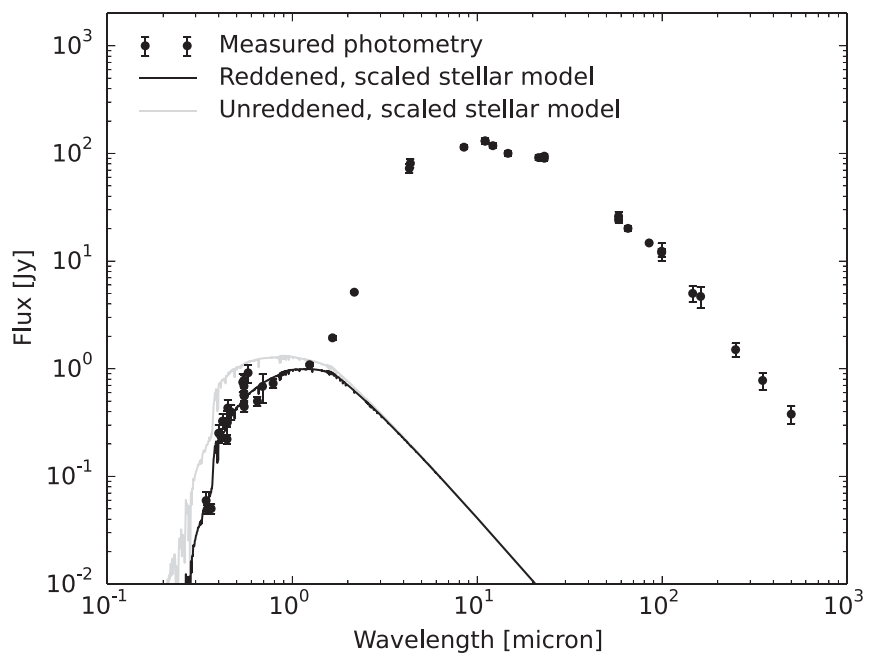

Figure 1. Photometry of AR Pup from the literature. A reddened and scaled stellar model photosphere and its dereddened version are overplotted. Note that this model does not illustrate the total stellar emission but rather represents the amount of scattered starlight observed at visible wavelengths (see Section 5.2 for a detailed discussion).

\section{Target Details}

AR Pup is a well-studied post-AGB binary system (Pollard et al. 1996; Hillen et al. 2017; Kiss \& Bódi 2017). The study by Pollard et al. (1996) showed that it is a RV Tauri ${ }^{15}$ variable of the photometric subclass $\mathrm{RVb}$. It has a pulsation period of 76.66 days and a RVb type modulation period of 1194 days (Kiss \& Bódi 2017). The latter phenomenon is commonly attributed to variable scattering and/or line of sight extinction of the starlight due to the binary's orbital motion (Waelkens et al. 1991, 1996) and thus constrains its orbital period (R. Manick et al. 2019, in preparation). The system's distance is $\sim 1$ to 6 kpc (van Leeuwen 2007; Gaia Collaboration 2018) and thus representative of nearby post-AGB stars (de Ruyter et al. 2006). AR Pup has been classified as a G-type star based on the spectroscopically derived stellar parameters from Gonzalez et al. (1997), who find $T_{\text {eff }}=6000 \mathrm{~K}, \log g=1.5$ (cgs system), and a $[\mathrm{Fe} / \mathrm{H}]=-1.0$. A detailed chemical abundance analysis has shown that AR Pup displays a photospheric chemistry depleted of refractory elements and is considered to be a strongly depleted post-AGB star (Gezer et al. 2015).

In Figure 1 we show selected photometry of the AR Pup system (see Appendix A for a table), adding measurements from the Herschel SPIRE Point Source Catalog (Schulz et al. 2017) to the data analyzed in detail by Hillen et al. (2017). To guide the eye, we overplot an ATLAS9 model atmosphere (Castelli \& Kurucz 2004) for the stellar parameters of AR Pup, reddened with $E(B-V)=0.27$ and scaled to match the visible photometry. A visual inspection clearly reveals its disktype nature via the presence of a near-infrared excess, as typically seen for disk-type sources (Section 1). The disk-type SED-based classification is further confirmed by the position of AR Pup on the WISE color-color plot (Gezer et al. 2015). The system's variability is obvious from the scatter of the various measurements at similar wavelengths in the visible. Hillen et al. (2017) found that the total infrared luminosity is higher than

\footnotetext{
${ }^{15}$ RV Tauri stars are luminous, variable stars (Type II Cepheids) with alternating deep and shallow brightness minima. The RVb subclass exhibits an additional long-term variation.
} 
that derived from the dereddened optical fluxes. They concluded that the disk of AR Pup must be oriented close to edge-on, so that the visible component of the SED is likely dominated by scattering, with most direct starlight being blocked by the optically thick disk.

\section{SPHERE Data}

The focus of this work is on high-angular-resolution observations of AR Pup at visible and near-infrared wavelengths. These data were obtained with the extreme AO instrument SPHERE (Beuzit et al. 2008) using its cameras Zurich Imaging Polarimeter (ZIMPOL, Thalmann et al. 2008) in the $V$ and $I$ bands, Infra-Red Dual-beam Imaging and Spectroscopy (IRDIS, Dohlen et al. 2008) in the $H$ band, and the integral field spectrograph (IFS, Claudi et al. 2008) in the $Y$ to $J$ band. In this section we describe the observations and data reduction.

\subsection{Observations}

Observations were carried out on 2016 April 4 in service mode (project ID: 097.D-0385, PI: S. Ertel). Observing conditions were well suited for our high-angular-resolution imaging of the relatively bright target $(R \sim 9.5)$ with a seeing between 0 ". 8 and 1 !' 1 and thin cirrus. The ZIMPOL observations were executed first, followed by the IRDIS and IFS observations, carried out simultaneously in IRDIFS mode, which combines the two modes for simultaneous use. In both cases, the observations were paired with observations of a point-spread function (PSF) reference star (HD 74128 for ZIMPOL, observed after AR Pup, $R=9.2$, HD 75363 for IRDIS, observed before AR Pup, $R=7.9$ ). Both stars were chosen to have a similar brightness as AR Pup in the observing bands used so that the same observing setup (in particular exposure time) as for the science target could be used. They also have similar brightness in the $R$ band and are close to AR Pup in the sky, so that the AO performance on science target and reference was as similar as possible. All observations were carried out in pupil stabilized mode to ensure that aberrations were as stable as possible.

For the ZIMPOL observations of both science target and PSF reference star, a single nine-point dither pattern was executed. The number of exposures per dither point was 16 for the science target and 7 for the reference star. A detector integration time (DIT) of $4 \mathrm{~s}$ was used for science target and reference star, making sure the source counts were within the linearity regime. This resulted in a total of 144 exposures, with a total integration time of $576 \mathrm{~s}$ on the science target and 63 exposures with a total integration time of $252 \mathrm{~s}$ on the PSF reference star. The observations were performed simultaneously in the $\mathrm{V}\left(\lambda_{0}=554.0 \mathrm{~nm}, \Delta \lambda=80.6 \mathrm{~nm}\right)$ and N_I $\left(\lambda_{0}=816.8 \mathrm{~nm}, \Delta \lambda=80.5 \mathrm{~nm}\right.$ ) filters using the two detectors of ZIMPOL in classical imaging mode (no polarization information obtained). A field stop was unintentionally inserted during the observations, limiting the field of view (FoV) of our ZIMPOL observations to $1^{\prime \prime}$.

The IRDIS data were obtained in dual-band imaging mode in the $\mathrm{H} 2\left(\lambda_{c}=1588.8 \mathrm{~nm}, \Delta \lambda=53.1 \mathrm{~nm}\right)$ and $\mathrm{H} 3 \quad\left(\lambda_{c}=\right.$ $1667.1 \mathrm{~nm}, \Delta \lambda=55.6 \mathrm{~nm})$ filters simultaneously. The observing strategy was analogous to that for ZIMPOL. A 16-point dither pattern was used, with three and two exposures per point on the science target and PSF reference star, respectively, and a DIT of $16 \mathrm{~s}$. This results in a total of 48 exposures with a total exposure time of $786 \mathrm{~s}$ on the science target and 32 exposures with a total exposure time of $512 \mathrm{~s}$ on the PSF reference star.

IFS observations in the $Y$ to $J$ band were carried out in parallel with the IRDIS observations using the IRDIFS mode. A total integration time of $1024 \mathrm{~s}($ DIT $=32 \mathrm{~s}$, NDIT $=16$, $\mathrm{NEXP}=2)$ on the science target and $544 \mathrm{~s} \quad($ DIT $=32 \mathrm{~s}$, NDIT $=17, \operatorname{NEXP}=1$ ) on the reference star was used.

\subsection{Data Reduction}

Data reduction was performed using the SPHERE pipeline version 0.18.0 and our own Python scripts.

All ZIMPOL frames of both science and reference observations were preprocessed using the pipeline to extract the informative component of the two detector frames (cutting off prescan and overscan areas and removing every second row which is masked on the detector) and to determine the overscan bias levels. In the resulting frames, the overscan bias was subtracted and each odd pixel was averaged with the following pixel in a row, creating an effective pixel scale of 7.2 mas. Flat fielding and bad pixel correction were not deemed necessary, given the image quality and the dither pattern performed during the observations. The reference frames were then centered on the source position (the location of the peak brightness in our images of the target) with an accuracy of 0.1 pix and stacked. The science frames were centered, derotated, and stacked. For each science frame, the stacked reference image was duplicated and rotated by the same angle, so that stacking these rotated PSFs results in the same rotational smearing of the resulting reference PSF as the derotation of the science frames.

Finally, the resulting science image was deconvolved with the reference PSF using the Richardson-Lucy deconvolution (Richardson 1972; Lucy 1974), which converged within 100 iterations. The results of our ZIMPOL data reduction are shown in Figure 2.

Master background and flat field frames for the IRDIS data were created using the pipeline and the observatory provided calibration frames. These frames were used to perform standard dark subtraction and flat fielding. In the resulting frames, bad and hot pixels were corrected using a median filtered map of each frame and a sigma clipping approach. Finally, to further reduce large scale detector cosmetics (stripes), we subtracted from each column and row its respective median in regions where no significant source flux was present. The frames still contained two images of the source-one for each filter. They were then cut to produce one frame per filter, and these frames were centered on the source. From here the reduction was performed analogous to the ZIMPOL reduction. Reference frames were stacked, science frames were derotated and stacked, the reference frames were duplicated and rotated the same way as the science frames, and the results were combined to produce a rotationally smeared reference PSF. The counts in the H3 filter are by factors of $\sim 2$ and $\sim 2.5$ larger for the calibrator and science target, respectively, compared to the $\mathrm{H} 2$ filter. Thus, combining the two images did not improve the signal-to-noise ratio $(\mathrm{S} / \mathrm{N})$ compared to using the $\mathrm{H} 3$ filter alone, and we used only the $\mathrm{H} 3$ data for the following image analysis.

The reference PSF was used to deconvolve the science image using the Richardson-Lucy deconvolution, which 


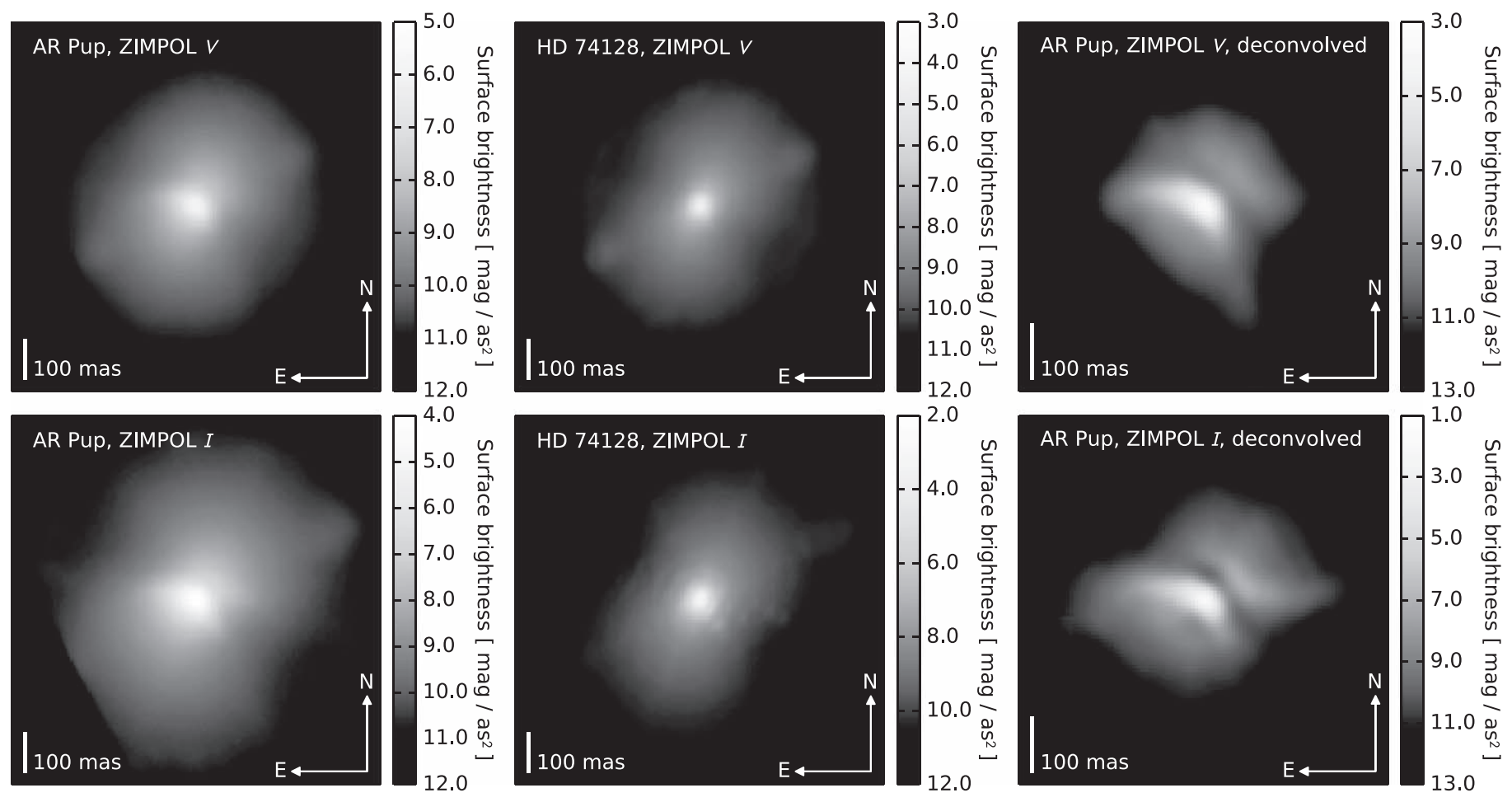

Figure 2. ZIMPOL observations of AR Pup (left), the PSF reference star HD 74128 (center), and the deconvolved images of AR Pup (right) in $V$ (top) and $I$ bands (bottom).

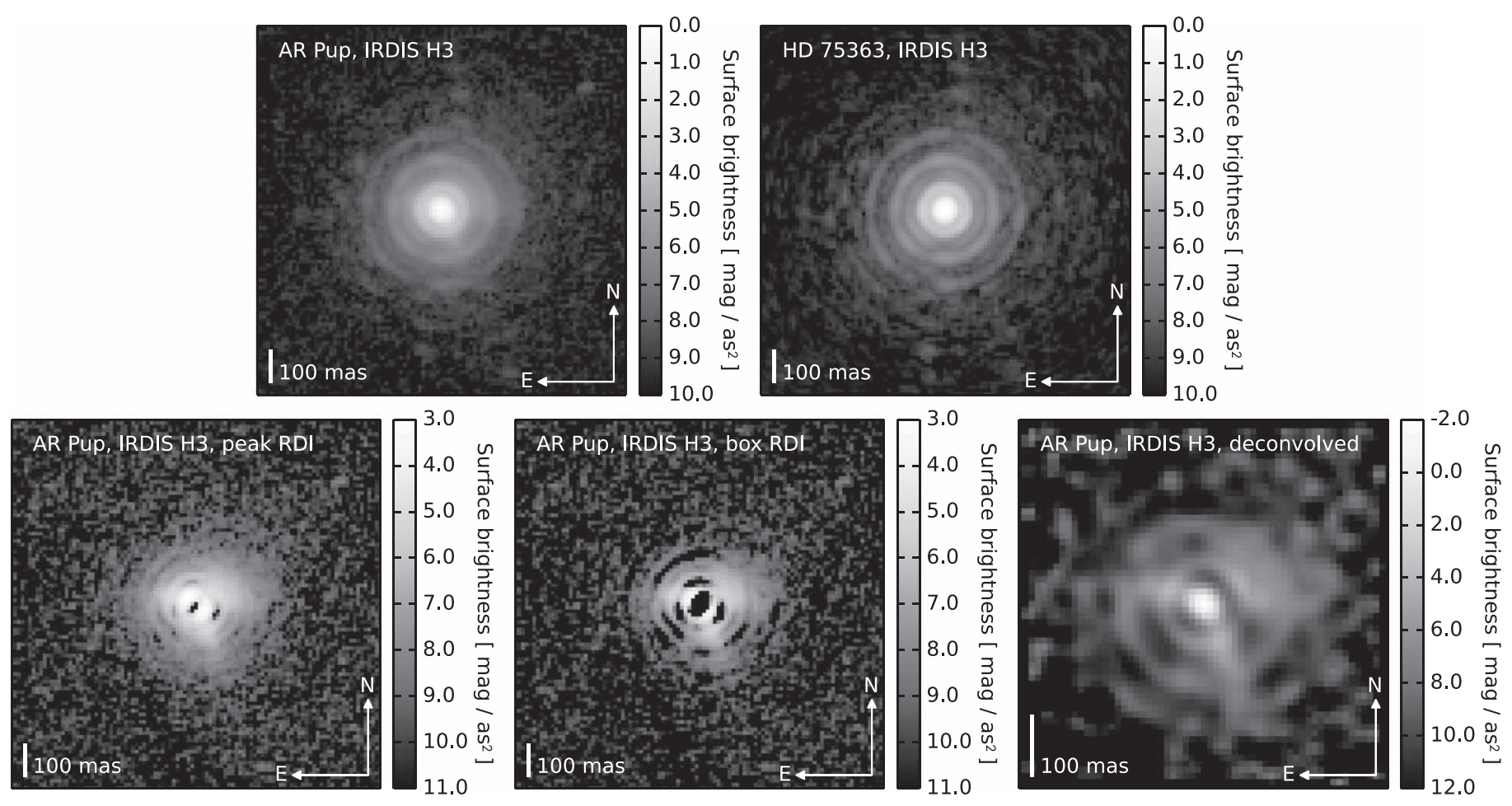

Figure 3. IRDIS observations of AR Pup (top left), the PSF reference star HD 75363 (top right), and the RDI subtracted and deconvolved images of AR Pup in the H3 filter (bottom row). Both approaches for the RDI are shown, where for "peak RDI" the reference star image was scaled to the same peak counts as the AR Pup image and for "box RDI" it was scaled to have the same counts as the AR Pup image in a quadratic aperture centered on the peak.

converged within 50 iterations. In addition, we performed reference star differential imaging (RDI) on the IRDIS data. This is difficult, because the bright central component of the science target is at least marginally extended. As a consequence, our reference PSF obtained by observing a point-like star is not an ideal representation of the flux distribution in our 
science image. Scaling the PSF to the peak counts of the science target and subtracting it reveals the extended core of the science target, but results in imperfect removal of the core's PSF from its surroundings. To reach a better contrast further away from the peak emission, we scaled the reference PSF to the same source counts as the science target in a squared $40 \times 40$ pixel box (equivalent to $490 \times 490$ mas, smaller than the AO control radius of 800 mas). This results in a strong oversubtraction of the core brightness, but a better removal of the core emission from its surroundings. In both cases, the subtraction of the reference PSF leaves strong residuals due to imperfect removal of the Airy rings, which are more blurred for the science target due to its extended core. The reduced IRDIS images are shown in Figure 3.

For the IFS data, the pipeline was used to create master background and flat field frames, a bad pixel map, and to determine the wavelength calibration from the observatory provided calibration frames as well as to extract the spectral image cubes. We then reduced the data for each spectral bin analogous to the IRDIS data. The source structures in the resulting image are consistent with those in the ZIMPOL and IRDIS images. However, the $\mathrm{S} / \mathrm{N}$ in these data is significantly worse than in the IRDIS data. It is dominated by residuals from the data reduction, likely due to cross-talk, such that combining multiple channels does not result in significant improvements. We thus do not further consider these data in the present work.

\subsection{Photometric Calibration}

We performed the photometric calibration of our images using the observed PSF reference stars as photometric calibrators. Aperture photometry of the IRDIS data was performed on each single frame. From the scatter of the counts from those measurements, we estimated an uncertainty of $5 \%$. We further add in quadrature a conservative uncertainty of $10 \%$, since with one calibrator observation we are not able to correct for various systematics related to air mass differences, variable transmission (thin cirrus during the observations), chromaticism, and so forth. We find magnitudes of AR Pup of $6.4 \pm 0.2 \mathrm{mag}$ and $6.2 \pm 0.2 \mathrm{mag}$ in the $\mathrm{H} 2$ and $\mathrm{H} 3$ filters, respectively, which is consistent within the uncertainties with the 2MASS $H$ magnitude of $6.824 \pm 0.042 \mathrm{mag}$ (Skrutskie et al. 2006) and the object's red near-infrared color $\left(J=7.891 \pm 0.029 \mathrm{mag}, K_{s}=5.285 \pm 0.020 \mathrm{mag}\right.$, not considering variability, ${ }^{16}$ Skrutskie et al. 2006). We used our derived magnitude measurements to calibrate the surface brightness of the extended emission.

Performing photometry on the ZIMPOL data is complicated by the small FoV and the extended halo visible in both the science and reference observations. This cannot be distinguished from a halo around the source expected from imperfect AO correction and thus has to be considered instrumental. No such halo is visible in the IRDIS images, which is also consistent with an AO artifact, in which case its intensity would scale with the inverse of the squared wavelength. We could assume that the fraction of emission in the halo is the same for the observations of science and reference target, and thus calibrate the total magnitude of our science target. However, to calibrate the surface brightness we need the total source counts.

\footnotetext{
16 2MASS observations in the $J, H$, and $K_{s}$ band were obtained simultaneously, so that colors derived from these measurements are not affected by temporal variability between measurements in single bands.
}

We thus separated the core and halo emission and approximated the halo with a circular Gaussian. From this, we estimated the total counts in the halo (inside and outside the FoV) and the total source counts. These could then be used to calibrate the magnitude of AR Pup and the surface brightness of the extended emission. We find that about $45 \%$ and $35 \%$ of the emission are in the halo in the $V$ and $I$ bands, respectively, and $50 \%$ of the halo emission is outside the FoV in both bands. The counts from different frames suggest an uncertainty of about $10 \%$. We conservatively add in quadrature $25 \%$ uncertainty, which is the fraction of total flux that we estimate to be outside the FoV. The resulting brightness of AR Pup is estimated to be $10.1 \pm 0.3 \mathrm{mag}$ and $9.0 \pm 0.3 \mathrm{mag}$ in the $V$ and $I$ bands, respectively. This compares to a $V$ magnitude of 9.6 at the time of the SPHERE observations estimated from our variability monitoring analysis (Section 5.2). Given the large uncertainties of SPHERE photometry, these measurements are consistent with the literature values. The $\sim 2 \sigma$ difference is on the high end of what can be expected by random measurement errors, and the fainter ZIMPOL measurement may suggest that we underestimate the emission in the extended halo. This illustrates the difficulties of performing accurate absolute photometry on AO data, in particular with a very small FoV.

\section{Imaging Results}

AR Pup's disk is only the second bona-fide post-AGB binary disk that has successfully been imaged in the visible and nearinfrared. Thus the disk geometry and the system's spatial features inferred from our images provide important insights into not only the AR Pup system but also the whole class of objects.

\subsection{General Morphology}

The reduced images from our observations of AR Pup and the reference stars are shown in Figures 2 and 3 for ZIMPOL and IRDIS, respectively. The stacked ZIMPOL images in both filters show a bright core that is elongated in the northeast to southwest direction. The same elongation can be surmised in the IRDIS data. Deconvolution of the ZIMPOL images reveals a clear "double-bowl" structure separated by a dark layer band. This is similar to the appearance of edge-on protoplanetary disks. We thus interpret the dark layer band as the optically thick disk mid-plane and the two "bowls" as the two disk surfaces scattering the starlight. We see no indication of direct stellar light reaching us through the disk, while the higher resolution images in $V$ and $I$ band provide stronger constraints than the $H$ band image. In the $V$ and $I$ bands we see no indication of direct stellar light reaching us through the disk (no central point source is visible). In $H$ band the lower angular resolution prevents us from drawing a firm conclusion, but the elongated core of the emission in Figure 3 suggests that transmitted starlight only contributes a small fraction to the total brightness. Ancillary optical long baseline interferometric data from the PIONIER at the VLTI show that the contribution of direct stellar light can be at most $10 \%$ of the total source brightness in the $H$ band (Appendix B).

The southeastern disk surface is brighter than the northwestern one. This suggests that the southeastern surface is oriented toward us, so that the starlight scattered on this surface reaches us directly. The light scattered on the northeastern disk surface is partly blocked by the disk following this 


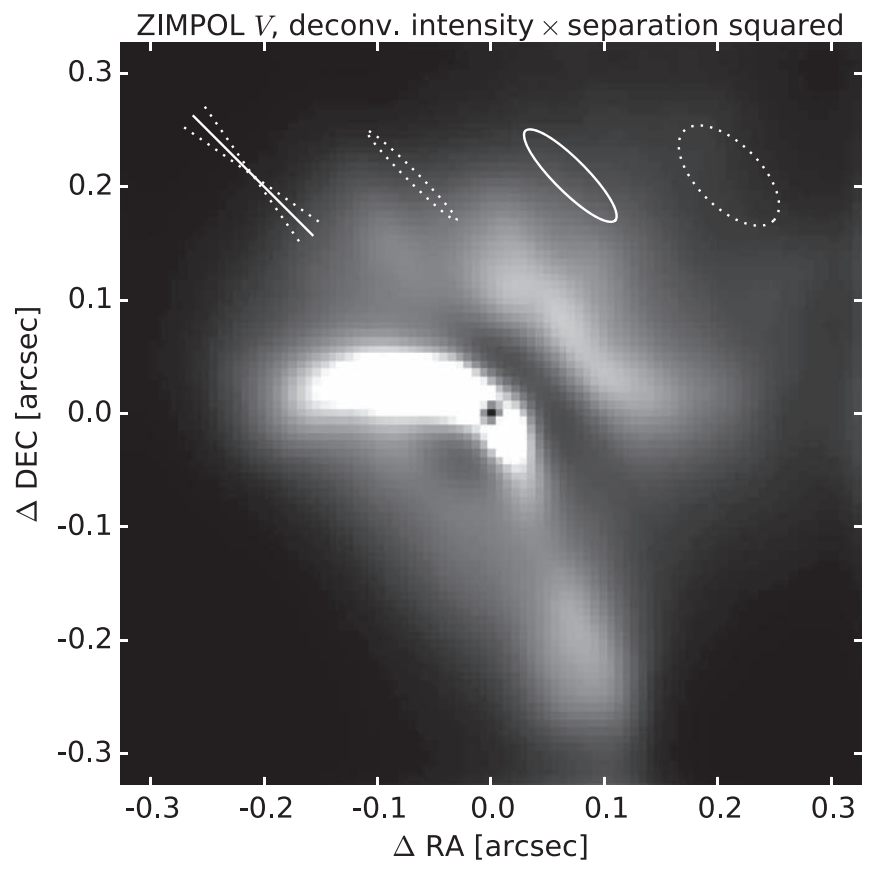

Figure 4. Analysis of the disk's position angle and inclination. The counts in the deconvolved $V$ band image have been multiplied by the squared projected separation from the peak location to approximately correct for the geometric dilution of starlight before being scattered by a dust grain. The solid line and ellipse illustrate the most plausible position angle and inclination, while the dashed lines and ellipse illustrate the plausible ranges.

interpretation. The curvature of the disk mid-plane also supports this view. The same structures are visible in the IRDIS data after deconvolution or PSF subtraction, albeit less clear due to the lower angular resolution and stronger residuals from the post-processing. The described features are the clearest in the deconvolved $I$ band image despite the nominally lower resolution compared to the $V$ band, which we attribute to a better AO correction at longer wavelengths. This is supported by the images of the reference star, where Airy rings are marginally visible in the $I$ band, but not in the $V$ band. The dark disk mid-plane has a radius of $\sim 50$ mas, while bright structures extend up to 300 mas from the disk center in the deconvolved ZIMPOL images. The size in our IRDIS images is somewhat smaller, most likely because the higher noise and residuals from PSF subtraction or deconvolution begin to dominate at smaller separations compared to the ZIMPOL images. The extended halo in the ZIMPOL images has to be attributed to imperfect AO correction, as discussed in Section 3.3.

\subsection{Disk Orientation}

We estimate the disk inclination and position angle from the deconvolved $V$ band image, which provides the highest angular resolution. To compensate approximately for the geometric dilution of the starlight before it is scattered by a dust grain at a given distance form the star, we multiply the counts in the images by the square of the projected separation from the peak location of the emission (the approximate center of the source). This further highlights the disk mid-plane. The result is shown in Figure 4. We then qualitatively fit ellipses to the mid-plane to assess the plausible ranges for the inclination and position angle of the disk. We estimate a disk inclination of $75_{-15}^{+10 \circ}$ from face-on and a position angle of the major axis of $45^{\circ} \pm 10^{\circ}$ east of north. A more formal analysis requires detailed radiative transfer modeling due to the complex interplay of disk orientation, scale height, flaring, optical depth, and scattering phase function of the dust, which is beyond the scope of this paper.

\subsection{Disk Substructure}

In addition to the global features of the disk image discussed in the previous sections, there are a number of arc-like substructures visible in both deconvolved ZIMPOL images and in part in the plain non-deconvolved ZIMPOL and deconvolved and PSF subtracted IRDIS images. We highlight the structures visible in the disk in Figure 5. To further illustrate the discussed features, we show in Figure 6 azimuthal profiles of the images in the two ZIMPOL filters at a separation of 175 mas ( 25 pixels) from the location of the peak brightness. The dark mid-plane is marked as feature A. The southeastern bowl (feature B) has a sharp, bright, arc-like contour, which could be caused by a combination of geometric and optical effects (viewing angle along the surface of the disk oriented toward Earth, forward scattering of the light on large dust grains compared to the observing wavelength). The brightest part of the arc shows a strong asymmetry and seems more extended toward the east in both ZIMPOL images. This could be explained by actual disk asymmetry or by an illumination effect due to the offset of the bright post-AGB star from the disk center on the binary orbit (see Section 5.2.2 for a more detailed discussion). Toward the south, the arc extends into a long, fainter arm in the ZIMPOL $V$ band and IRDIS images. In the ZIMPOL $I$ band image, the faint arc seems similarly extended toward the south, albeit less obviously so. However, the arc appears fainter in the $I$ band, so that it partly blends with the rest of the disk emission. This explains why the southern peak B in Figure 6 is only visible in the $V$ band profile, not the $I$ band.

The northwestern bowl shows multiple, arc-like substructures in the ZIMPOL images (features C). These structures also have tentative counterparts in the deconvolved IRDIS image, although they cannot be well distinguished from deconvolution residuals. They are weak modulations of the surface brightness and are not obvious in the plain non-deconvolved images. Their locations seem anti-correlated with the directions of two PSF features best seen in the $I$ band reference star image (beams in both directions of the PSF peak at position angles of $\sim 120^{\circ}$ and $\sim 160^{\circ}$ East of North). It is possible that these features were not entirely stable during the observations of science target and reference star. This could have been overcompensated for during the deconvolution process, resulting in dark artifacts at the location of the PSF features, thus causing apparently bright features at other locations. However, the PSF features are symmetric around the core of the PSF. The fact that the supposed disk features are only visible on one side makes this scenario unlikely.

Understanding the origin of these arc-like features in the northwestern side of the disk can give important insights into the disk architecture, the system's evolution, and binary-disk interaction. Kervella et al. (2015) observed similar features in their images of the $\mathrm{L}_{2}$ Pup disk (dubbed plumes in their paper). They suggested the features originate in an outflow of 

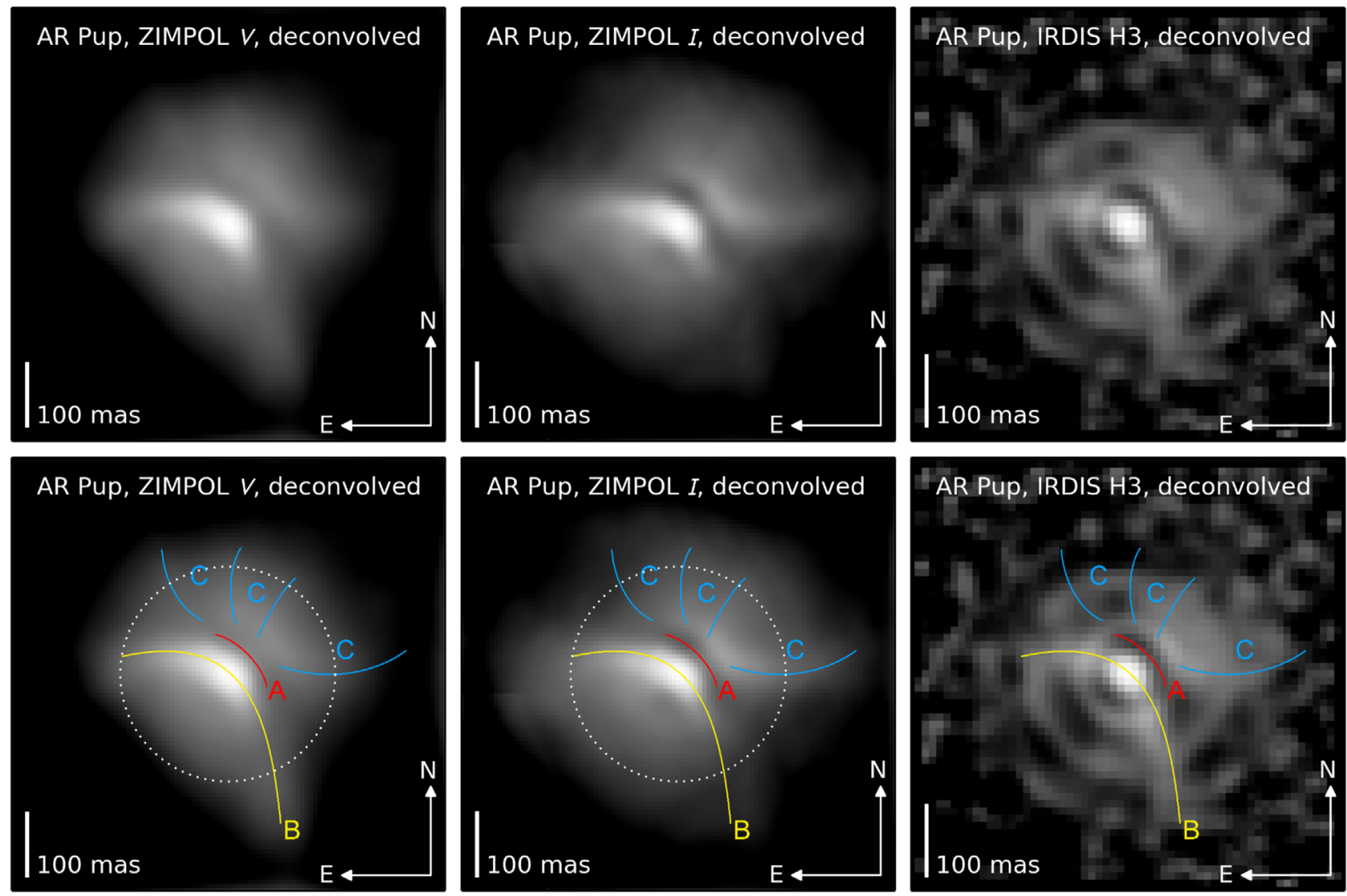

Figure 5. Disk morphology and structures. The deconvolved ZIMPOL $V$ (left) band, ZIMPOL $I$ band (center), and IRDIS (right) images are shown in the top row for clarity. The same images are shown in the bottom row, with overlays highlighting the disk features discussed in Section 4.3. The dotted circles mark the separation at which the azimuthal profiles in Figure 6 have been extracted.

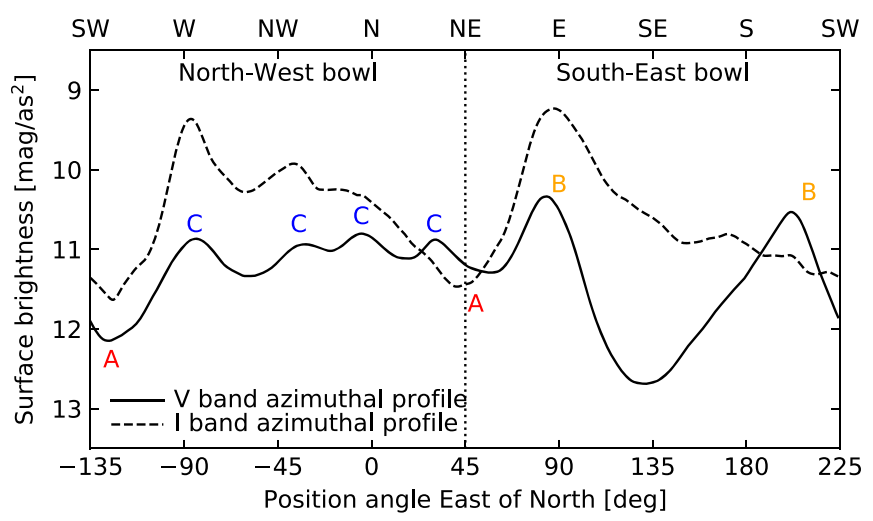

Figure 6. Azimuthal profiles of the images in the two ZIMPOL images at a separation of 175 mas ( 25 pixels) from the location of the peak brightness (marked by the dotted circles in Figure 5). The letters A, B, and C mark the locations of the disk features discussed in Section 4.3 and shown in Figure 5. Uncertainties on the profiles are dominated by systematics from deconvolution and likely considerable (see Section 4.3 for details).

material from the binary star (wind or jet, as observed, e.g., by Bollen et al. 2017 in the BD+46 442 system). An outflow in the form of a disk wind as has been observed by Bujarrabal et al. (2018) for IRAS 08544-4431 would be another possible explanation. If the features are indeed caused by outflows, they could trace the processes producing the bipolar morphologies observed in more evolved protoplanetary nebulae and planetary nebulae.

Similar scattered light features have been observed in young protoplanetary disks (Casassus et al. 2012; Stolker et al. 2016; Benisty et al. 2017). These data reveal both spirals in the outer disk and structures in the inner disk regions casting shadows onto the outer disk. Price et al. (2018) modeled the binary-disk interaction for the circumbinary disk HD 142527 and were able to reproduce all observed features. These studies can inform similar studies of the features seen in the AR Pup and $\mathrm{L}_{2}$ Pup images.

Monitoring the time evolution of the features can help establish their nature (Debes et al. 2017): a slow evolution on the orbital timescale of material in the outer disk would suggest actual structures in the outer disk. Faster evolution on the orbital timescale of the disk material close to the star would suggest a shadowing effect. An outflow from the binary is expected to cause a modulation of the features on the binary orbital timescale.

\section{Discussion}

\subsection{Comparison to Other Disks around Evolved Stars Imaged in Scattered Light}

The Red Rectangle is indubitably the case most similar to AR Pup of a disk imaged around an evolved star (Osterbart et al. 1997; Cohen et al. 2004). The disk around the Red 
Rectangle's post-AGB RVb binary central star (Waelkens et al. 1996) could be imaged with the $H S T$ due to its larger angular size of $\sim 1^{\prime \prime}$. It can be explained by a closer distance $(\sim 500 \mathrm{pc}$, van Leeuwen 2007), a larger physical disk size, or a combination of both. This disk shows a similar overall shape with a double-bowl structure and a dark mid-plane, but the two bowls show a weaker brightness asymmetry, suggesting that the disk is seen closer to edge-on. The primary is of spectral type B9, suggesting that it is more evolved than AR Pup. The system shows extended red emission, which we would be unable to detect in our data of AR Pup due to our small FoV (ZIMPOL) and limited sensitivity to faint, extended emission (IRDIS). No Hubble images of AR Pup are available.

The disks around $\mathrm{L}_{2}$ Pup (Kervella et al. 2015) and BP Psc (Zuckerman et al. 2008; de Boer et al. 2017) are other potentially similar systems, albeit less clear: $\mathrm{L}_{2}$ Pup has been characterized as a nearby AGB star $(d=64 \mathrm{pc}$, van Leeuwen 2007) with a planetary mass companion (Kervella et al. 2016; Homan et al. 2017), and its disk is more compact with a size of $\sim 25$ au. The distance of BP Psc has recently been measured by Gaia to be $\sim 350$ pc (Gaia Collaboration 2018), confirming its nature as a first ascent giant star (Zuckerman et al. 2008). The disk has a diameter of $\sim 35$ au $(\sim 100$ mas at $\sim 350 \mathrm{pc})$, similar to that of $\mathrm{L}_{2}$ Pup. Neither $\mathrm{L}_{2}$ Pup nor BP Psc are known to show long-term variability that could be attributed to binary orbital motion.

If the planetary mass companion of $\mathrm{L}_{2}$ Pup was responsible for the formation of the disk, it cannot have delivered as much angular momentum as the stellar mass companion of AR Pup. This would explain the smaller disk around $\mathrm{L}_{2}$ Pup and the fact that the star is still characterized as an AGB star (i.e., the interaction with the companion that created the disk did not terminate the star's AGB phase). The disk size and evolutionary state of BP Psc as a first ascent giant star (Zuckerman et al. 2008) suggest that for this star, too, a companion of planetary mass - rather than stellar mass - may be responsible for the disk formation.

Given their small angular sizes compared to the angular resolution of the instrument with which they were imaged, it is no surprise that all four disks are seen close to edge-on, at least partly obscuring their host stars. Imaging disks oriented closer to face-on requires more sophisticated high-contrast techniques to reveal circumstellar disks of similar size, such as polarimetric differential imaging. Such observations of postAGB disks have the potential to provide more insight into the disk properties, as they better allow us to image radial and azimuthal brightness variations of the disk surface more directly.

\subsection{Connection to Other Observables of the System}

We have demonstrated that it is now possible with the newest extreme AO systems to image disks around post-AGB binaries. This is, however, typically still only possible for systems within a few kpc, depending on linear disk size. Furthermore, AR Pup is particularly well suited for this due to its edge-on disk masking the bright stellar light, thus reducing the required image contrast. Spatially resolved imaging of a few benchmark systems such as AR Pup and connecting other observables of these systems to the properties of the disks are vital to our understanding of systems that cannot be resolved. Here we briefly discuss AR Pup's SED and photometric variability in the light of our SPHERE images. A more comprehensive analysis will be enabled by imaging a sample of post-AGB binary disks.

\subsubsection{Spectral Energy Distribution}

Hillen et al. (2017) found from their study of AR Pup's SED that the total infrared luminosity is higher than that derived from the dereddened optical fluxes. They suggest that the disk of AR Pup must be seen close to edge-on, so that most direct starlight is blocked. We confirm this conclusion and expand on it with our resolved images of the system, showing that the light seen at visible wavelengths stems entirely from starlight scattered in our direction by the dust on the disk surface. The derived value of $E(B-V)$ has thus to be understood as the result of a combination of effects (Scicluna \& Siebenmorgen 2015): the light is altered by scattering events in the disk and affected by circumstellar and interstellar extinction on its indirect path from the star toward us. The measured $E(B-V)$, however, heavily underestimates the circumstellar extinction on the direct line of sight from the star. Thus, the unreddened stellar model in Figure 1 heavily underestimates the total stellar emission, so that it cannot be used to determine the stellar luminosity or the star's distance if the luminosity is estimated or derived independently.

At near-infrared and longer wavelengths we start seeing the thermal emission of the disk. The onset of this emission around the $H$ band (Appendix B) indicates that the hottest material has a temperature of $\sim 1200 \mathrm{~K}$. The bulk emission peaks around $10 \mu \mathrm{m}$, indicating a temperature around $\sim 550 \mathrm{~K}$ for the majority of the dust. Detailed radiative transfer modeling of our scattered light images and the SED can be used to derive accurate disk parameters such as geometry and dust properties.

\subsection{2. $R V b$ Phenomenon}

The fact that no direct stellar light is seen in the visible also has consequences for the interpretation of the system's observed $\mathrm{RVb}$ variability. To accurately determine the phase of the variability during our SPHERE observations, we complement photometric time series data from the All Sky Automated Survey (ASAS, Pojmanski 2002) with own photometric monitoring observations between 2016 March 17 and 2016 June 9 (around the time of our SPHERE observations). Our observations were performed at Mt. Kent Observatory (near Toowoomba, Queensland, Australia) using a PlaneWave CDK700 telescope equipped with an Alta U16M Apogee camera. Data reduction and photometric analysis were performed using the AstroImageJ software package (Collins et al. 2017). We analyze the ASAS and Mt. Kent data following Manick et al. (2017) and recover the 76.66 days pulsation period and the 1194 days RVb period found by Kiss \& Bódi (2017) within the uncertainties. We find that the brightness of AR Pup was increasing and close to its average value during our SPHERE observations from both the pulsation variability ( $\sim 5$ days past minimum) and the long-term variability ( $\sim 300$ days past minimum). This is illustrated in Figure 7. Imaging the system at different phases of its variability will allow us to connect the photometric variation, variations in the disk features, and the orbital phase of the binary. 


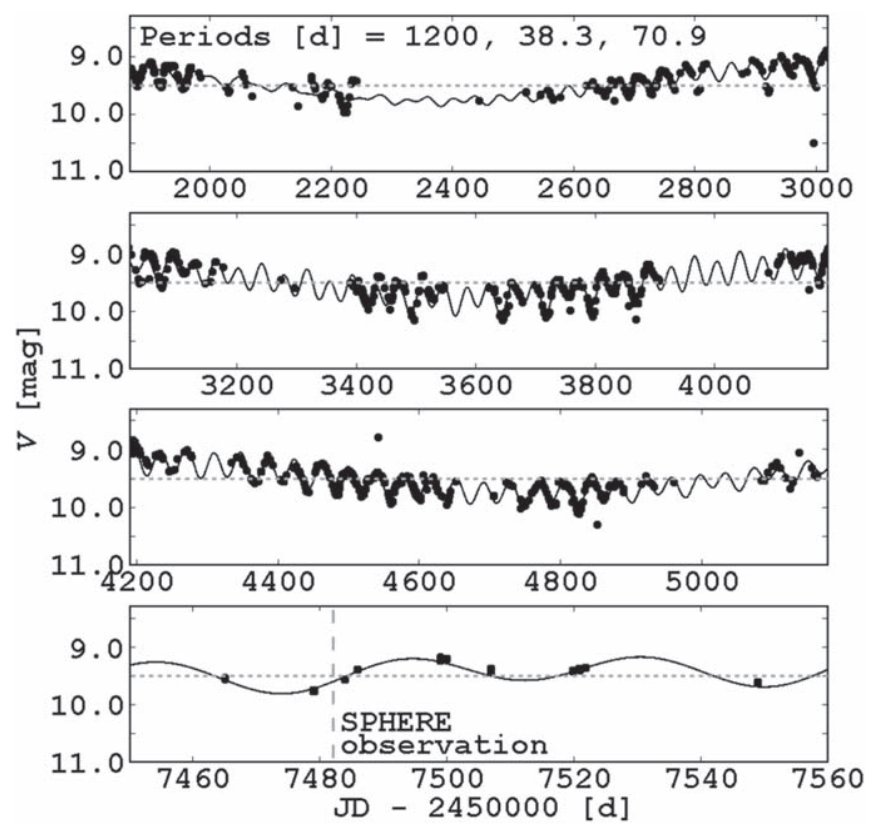

Figure 7. Photometric monitoring of AR Pup. The horizontal, dotted line indicates the approximate mean magnitude of $V=9.5$. The time of the SPHERE observations is highlighted by the vertical, dashed line.

The origin of the long-term variability of $\mathrm{RVb}$ stars has been attributed to either variable extinction or variable scattering of starlight by a circumbinary disk. For example, Waelkens et al. (1991) concluded for HR 4049 based on the color dependence of the variability that it likely stems from variable extinction. For the Red Rectangle, Waelkens et al. (1996) concluded that it must stem from variable scattering because no color variation was witnessed and the star is completely obscured by the edgeon disk. Kiss \& Bódi (2017) analyzed the amplitude variations of the short period variability of RVb stars over the long period and conclude that they are consistent with variable obscuration by the disk.

For AR Pup, we show that the star is fully obscured by the disk, similar to the Red Rectangle. The star was close to its mean brightness in both the long-term and short-term variability cycles, so we cannot simply see the moment of maximum obscuration over the cycle of variable extinction. Instead, the variability must be caused by variable scattering on the disk surface, similar to the Red Rectangle. We find the disk of AR Pup to be slightly inclined away from edge-on, and we see structures in the disk that may be attributed to illumination effects. We thus propose that variable disk illumination over the binary's orbital period is an alternative scenario (although the two are not mutually exclusive) to the variable scattering angle as the main source of the brightness variations proposed by Waelkens et al. (1996) for the Red Rectangle.

We illustrate in Figure 8 how variable illumination of the disk by the post-AGB star on its orbit can readily explain both the brightness variation and the asymmetric brightness of the disk in our images. We start with a disk that has a vertically thin inner edge. The side of the disk closer to the bright postAGB star on its orbit will be brighter than the other side. When the star is on the far side of its orbit, the far, visible side of the disk will be brighter, while the near side of the disk will be

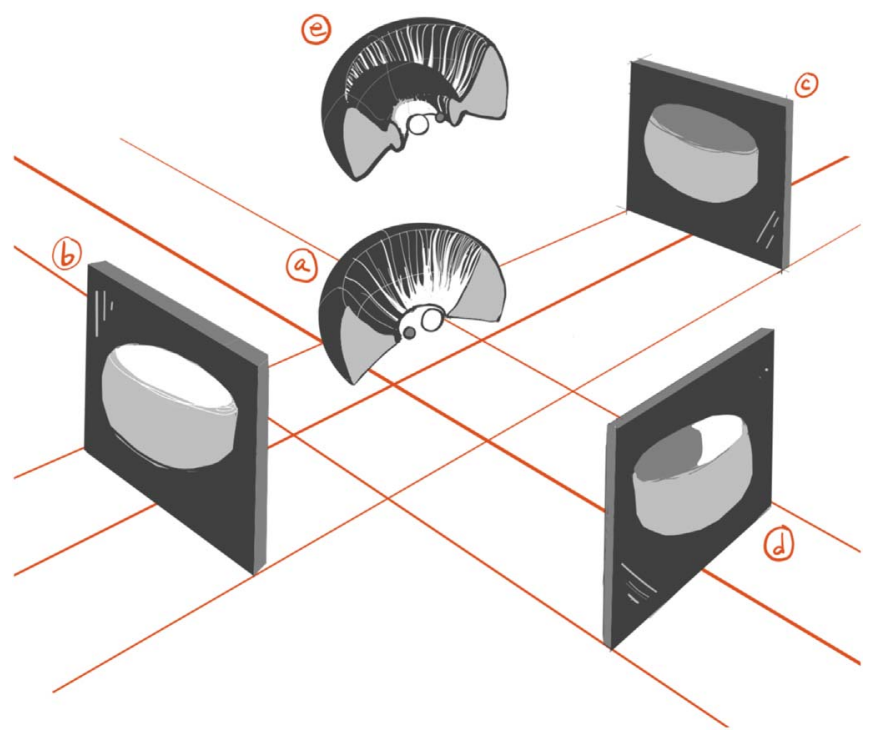

Figure 8. Illustration of the disk illumination effect producing the $\mathrm{RVb}$ phenomenon in the AR Pup disk. A disk with a vertically thin inner edge is shown (a), and its appearance when viewed from various orientations is illustrated: (b) appearance if the post-AGB star is on the far side of its orbit, (c) appearance if the post-AGB star is on the near side, and (d) appearance if the post-AGB star is on the right. A disk with an inflated inner edge is also shown (e), but the binary phase is inverted so that the appearance of the disk in panels (b), (c), and (d) is the same.

fainter (b). The system will thus be in a bright phase. As the post-AGB star moves to the near side on its orbit, the brightness of the system decreases as the visible side of the disk becomes fainter (c). During our SPHERE observations, the star was approximately halfway between the near and far sides on its orbit, in which case the disk would show a brightness asymmetry due to the way it is illuminated (d). An orbital period of 1194 days implies an orbital semimajor axis of $\gtrsim 1$ au. If the inner edge of the disk is plausibly at $5 \mathrm{au}$, then the star would be separated by $\gtrsim 4$ au from one side and $\gtrsim 6$ au from the other side of the disk, causing a difference in illumination by a factor $\gtrsim 2.25$. This is large enough to explain the long-term variability of the system. Alternatively, the inner rim of the disk may be inflated due to the heating from the star (e). This way, it would cast a shadow onto the side of the outer disk close to the post-AGB star. This side would then be fainter, having the opposite effect compared to the scenario with a vertically thin disk edge. The effect may be enhanced by the variable heating of the inner disk edge by the star on its orbit with a larger scale height close to the post-AGB star (Kluska et al. 2018). Disk structures created by the gravitational interaction with the binary star may play an additional role in the shadowing scenario.

These two scenarios demonstrate how resolved imaging in combination with detailed knowledge of the binary orbit can help us understand the structure of the disk and its interaction with the binary star. Unfortunately, for AR Pup a spectroscopic orbit is not available at the moment. Obtaining such an orbit is one of the critical next steps in the study of this system. In addition, multi-epoch imaging of the disk is important to monitor the variation of the brightness asymmetry. Comparing the timescale of this variability with the timescale of the long-term 
variability is a critical test of the two scenarios described previously.

\subsection{An Extreme Laboratory for Circumstellar Disk Evolution}

Several studies have now established the similarities between post-AGB binary disks and protoplanetary disks (de Ruyter et al. 2005; Gielen et al. 2011; Hillen et al. 2014, 2015, 2017, P. Scicluna et al. 2019, in preparation). Our results on AR Pup add to this picture by showing the similar morphology and scale of this disk from high fidelity images. In particular, the presence of highly processed grains up to millimeter size found by these works may come as a surprise, given the short disk lifetimes $\left(\sim 10^{4}\right.$ yr; Bujarrabal et al. 2018) and the fast evolution of their host stars $\left(10^{2}-10^{4} \mathrm{yr}\right.$, Miller Bertolami 2016; $10^{3}-10^{4} \mathrm{yr}$, Sahai et al. 2007; Gesicki et al. 2010). Only recently, Harsono et al. (2018) were able to infer the presence of millimeter-sized dust grains in the $10^{5} \mathrm{yr}$ young disk around the protostar TMC1A, while most protoplanetary disks are only observed at an age of $\gtrsim 10^{6} \mathrm{yr}$ (e.g., Hartmann et al. 1998). Post-AGB binary disks are thus interesting extreme laboratories to study circumstellar disk evolution and to constrain the timescales of dust grain growth, which is a critical step in the planet formation process.

Due to their short lifetime, it remains questionable if postAGB binary disks could actually form planets. However, large inner cavities such as the ones often attributed to the presence of planets in transition disks (although there may be other formation scenarios, Alexander et al. 2014; Turner et al. 2014) have been found in both the disks around the post-AGB binary AC Her (Hillen et al. 2015) and the Class I protostar WL 17 (age of a few $10^{5} \mathrm{yr}$ ) (Sheehan \& Eisner 2017). In particular, the presence of a few large bodies such as left-over planetesimals from the main sequence might act as a catalyst in post-AGB binary disks (Birnstiel et al. 2012, 2016; Garaud et al. 2013; Bitsch et al. 2015). The dust masses estimated by de Ruyter et al. (2005) seem consistent with those of protoplanetry disks (Andrews et al. 2013; Ansdell et al. 2016, 2017; Barenfeld et al. 2016).

Several other studies have suggested a variety of avenues for second-generation planet formation beyond a star's main sequence lifetime, suggesting this might not be an uncommon process. The claimed ${ }^{17}$ discoveries of planetary systems orbiting post-common envelope binary stars (e.g., Lee et al. 2009; Almeida et al. 2013; Pulley et al. 2018) are best explained by such a scenario (Mustill et al. 2013). The planets detected orbiting pulsars are another such example (Wolszczan \& Frail 1992; Wang et al. 2006). Recently, van Lieshout et al. (2018) proposed a scenario of how even Earth mass, rocky planets could form in the habitable zones around white dwarfs.

\section{Conclusions}

We have presented the first resolved images of the circumbinary disk around the post-AGB star AR Pup. Using VLT/SPHERE observations in the visible and image deconvolution with an observed reference star, as well as RDI, we were able to reveal circumstellar emission in the $V, I$, and $H$ bands. The disk is optically thick in all our images and seen

\footnotetext{
17 A number of these candidates have been shown to be dynamically unstable (e.g., Horner et al. 2011; Wittenmyer et al. 2013; Pulley et al. 2018), which potentially casts doubt on the existence of those claimed planets.
}

close to edge-on. The dark disk mid-plane has a radius of about 50 mas, and extended emission is detected out to a separation of about 300 mas from the source center. We estimate an inclination of $75_{-15}^{+10 \circ}$ from face-on and a position angle of the disk major axis of $45^{\circ} \pm 10^{\circ}$ east of north.

Several arc-like features are identified in the visible images. We discussed various scenarios to explain these features, including a disk wind, outflows or jets from the central binary due to stellar wind or accretion, and shadows cast onto the outer disk by disk structures closer in due to binary-disk interactions. Resolved images at various phases of the binary orbit can potentially allow us to distinguish between these scenarios.

There is no indication of direct stellar light in our visible images and likely neither in the $H$ band image. We thus conclude that the long-term $\mathrm{RVb}$ photometric variability of the system must be caused by variable scattering, not extinction, of starlight over the binary orbit. We propose a scenario of variable disk illumination, in addition to previously discussed variations of the scattering angle, to explain this variability.

Finally, we highlight the value of post-AGB binary disks as extreme laboratories to study circumstellar disk evolution. The high degree of dust processing observed in these disks, despite their short lifetimes of $<10^{5} \mathrm{yr}$, strongly constrains the timescales at which dust processing in disks can happen. If a connection to protoplanetary disks can be made, this constrains the swiftness of the early stages of the planet formation process. Along these lines, we argue that second-generation planet formation beyond a star's main sequence lifetime might not be uncommon.

S.E. acknowledges support through the ESO fellowship program and thanks C. Melo for his support and encouragement to pursue this research. G.H.M.B. acknowledges funding from the European Research Council (ERC) under the European Union's Horizon 2020 research and innovation programme (grant agreement No. 757957). This research has been supported by the Ministry of Science and Technology of Taiwan under grants MOST104-2628-M-001-004-MY3 and MOST107-2119-M-001-031-MY3, and Academia Sinica under grant AS-IA-106-M03. E.V. is supported by Spanish grant AYA 2014-55840-P. We have made use of the SIMBAD database, operated at CDS, Strasbourg, France, and NASA's Astrophysics Data System. Herschel is an ESA space observatory with science instruments provided by Europeanled Principal Investigator consortia and with important participation from NASA. This work benefitted from the FEARLESS collaboration (FatE and AfteRLife of Evolved Solar Systems, PI: S. Ertel).

Facility: VLT: Melipal (SPHERE).

Software: Python, Astropy (Astropy Collaboration et al. 2013), NumPy (Oliphant 2006), SciPy (Jones et al. 2001), and Matplotlib (Hunter 2007) libraries.

\section{Appendix A SED Data}

In Table 1 we list the photometry and corresponding references used to build the SED in Figure 1. 
Table 1

Photometry of AR Pup

\begin{tabular}{|c|c|c|c|c|}
\hline $\begin{array}{l}\text { Wavelength } \\
(\mu \mathrm{m})\end{array}$ & $\begin{array}{c}\text { Flux } \\
(\mathrm{Jy})\end{array}$ & $\begin{array}{l}\text { Uncertainty } \\
\text { (Jy) }\end{array}$ & $\begin{array}{l}\text { Instrument/ } \\
\text { Filter }\end{array}$ & References \\
\hline 0.34 & 0.060 & 0.012 & GENEVA & Mermilliod et al. (1997) \\
\hline 0.35 & 0.0495 & 0.0049 & STROMGREN & Hauck \& Mermilliod (1998) \\
\hline 0.36 & 0.0502 & 0.0050 & JOHNSON & Mermilliod et al. (1997) \\
\hline 0.40 & 0.252 & 0.049 & GENEVA & Mermilliod et al. (1997) \\
\hline 0.41 & 0.235 & 0.024 & STROMGREN & Hauck \& Mermilliod (1998) \\
\hline 0.42 & 0.326 & 0.053 & GENEVA & Mermilliod et al. (1997) \\
\hline 0.44 & 0.313 & 0.094 & USNOB1 & Monet et al. (2003) \\
\hline 0.44 & 0.317 & 0.032 & JOHNSON & Anderson \& Francis (2012) \\
\hline 0.44 & 0.222 & 0.022 & JOHNSON & Mermilliod et al. (1997) \\
\hline 0.44 & 0.323 & 0.010 & JOHNSON & Kharchenko et al. (2007) \\
\hline 0.45 & 0.432 & 0.084 & GENEVA & Mermilliod et al. (1997) \\
\hline 0.47 & 0.396 & 0.064 & STROMGREN & Hauck \& Mermilliod (1998) \\
\hline 0.54 & 0.75 & 0.15 & GENEVA & Mermilliod et al. (1997) \\
\hline 0.55 & 0.781 & 0.097 & GENEVA & Mermilliod et al. (1997) \\
\hline 0.55 & 0.691 & 0.069 & STROMGREN & Mermilliod et al. (1997) \\
\hline 0.55 & 0.573 & 0.093 & STROMGREN & Hauck \& Mermilliod (1998) \\
\hline 0.55 & 0.444 & 0.044 & JOHNSON & Mermilliod et al. (1997) \\
\hline 0.55 & 0.562 & 0.014 & JOHNSON & Kharchenko et al. (2007) \\
\hline 0.55 & 0.559 & 0.056 & JOHNSON & Anderson \& Francis (2012) \\
\hline 0.58 & 0.92 & 0.18 & GENEVA & Mermilliod et al. (1997) \\
\hline 0.65 & 0.499 & 0.050 & COUSINS & Anderson \& Francis (2012) \\
\hline 0.69 & 0.69 & 0.21 & USNOB1 & Monet et al. (2003) \\
\hline 0.79 & 0.732 & 0.073 & COUSINS & Anderson \& Francis (2012) \\
\hline 1.24 & 1.090 & 0.029 & 2MASS & Skrutskie et al. (2006) \\
\hline 1.65 & 1.936 & 0.075 & 2MASS & Skrutskie et al. (2006) \\
\hline 2.16 & 5.140 & 0.095 & 2MASS & Skrutskie et al. (2006) \\
\hline 4.29 & 72.7 & 6.8 & $M S X$ & Egan et al. (2003) \\
\hline 4.35 & 81.0 & 7.5 & MSX & Egan et al. (2003) \\
\hline 8.48 & 114.3 & 4.7 & $M S X$ & Egan et al. (2003) \\
\hline 11.0 & 129.9 & 5.3 & IRAS & Moshir et al. (1990) \\
\hline 11.0 & 131.0 & 7.9 & IRAS & Helou \& Walker (1988) \\
\hline 12.2 & 118.0 & 5.9 & $M S X$ & Egan et al. (2003) \\
\hline 14.7 & 100.2 & 6.1 & MSX & Egan et al. (2003) \\
\hline 21.5 & 91.5 & 5.5 & $M S X$ & Egan et al. (2003) \\
\hline 23.1 & 90.2 & 4.3 & IRAS & Moshir et al. (1990) \\
\hline 23.1 & 94.3 & 4.7 & IRAS & Helou \& Walker (1988) \\
\hline 58.2 & 24.3 & 1.5 & IRAS & Moshir et al. (1990) \\
\hline 58.2 & 26.1 & 2.6 & IRAS & Helou \& Walker (1988) \\
\hline 65.4 & 20.13 & 0.92 & AKARI & Murakami et al. (2007) \\
\hline 85.1 & 14.74 & 0.28 & AKARI & Murakami et al. (2007) \\
\hline 99.5 & 12.0 & 1.1 & IRAS & Helou \& Walker (1988) \\
\hline 99.5 & 12.4 & 2.2 & IRAS & Moshir et al. (1990) \\
\hline 146 & 5.012 & 0.85 & AKARI & Murakami et al. (2007) \\
\hline 162 & 4.7 & 1.1 & AKARI & Murakami et al. (2007) \\
\hline 250 & 1.610 & 0.083 & SPIRE & (Schulz et al. 2017) \\
\hline 350 & 0.861 & 0.099 & SPIRE & (Schulz et al. 2017) \\
\hline 500 & 0.429 & 0.073 & SPIRE & (Schulz et al. 2017) \\
\hline
\end{tabular}

\section{Appendix B PIONIER Observations}

PIONIER (Le Bouquin et al. 2011) observations of AR Pup were carried out on 2015 February 24 (Project 094.D-0865, PI: Hillen) using the compact array configuration of the Auxiliary Telescopes of the VLTI (interferometric baselines between 11.3 and $34.3 \mathrm{~m}$ ). Fringes could only be detected at the shortest baseline (projected baseline changing from 8.9 to $9.1 \mathrm{~m}$ over the 10 minute sequence of exposures). Five sequences of 100 scans each were recorded in six spectral channels across the $H$ band, resulting in $5 \times 6=30$ squared-visibility $\left(V^{2}\right)$ measurements. HD 67556 was observed after AR Pup as a calibration star in the same instrument setup. Data were reduced using version 3.30 of the PIONIER pipeline. The squared-visibility measurements are shown in Figure 9.

The bright central star of AR Pup is expected to be unresolved at short baselines (diameter $<1$ mas), and the contribution of the faint companion is negligible, so that the stellar $V^{2}$ is expected to be one. The fact that fringes could only be detected at the shortest baseline and the low visibility suggest that the source is extended. We show in Figure 9 that a Gaussian source brightness distribution with a full width at half maximum (FWHM) of 25 mas reproduces the data well.

In order to put an upper limit on the amount of direct starlight contributing to the total source emission in $H$ band, we add to the Gaussian brightness distribution an unresolved point 


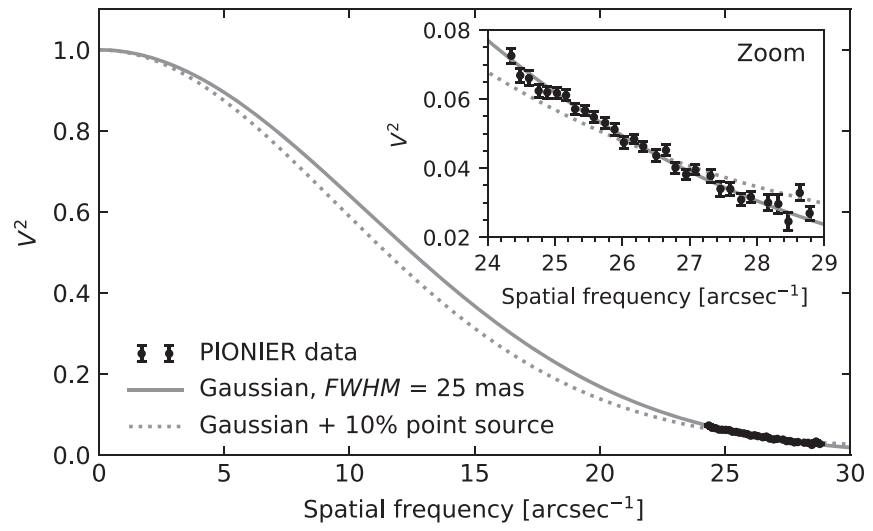

Figure 9. PIONIER squared-visibility measurements of AR Pup in $H$ band at a projected changing from 8.9 to $9.1 \mathrm{~m}$ over the 10 minute sequence of exposures. Overplotted are a Gaussian source brightness distribution with $\mathrm{FWHM}=25$ mas and a model consisting of a Gaussian (FWHM = 29 mas) plus a point source contributing $10 \%$ to the total source flux.

source (the star) following, for example, di Folco et al. (2007). No satisfactory fit with a substantial amount of direct starlight could be achieved. We show in Figure 9 a model with a $10 \%$ point source and an FWHM of the Gaussian component of 29 mas, which produces a mediocre fit to the data. We thus put a conservative upper limit of $10 \%$ on the contribution of direct starlight to the total source emission. The fact that no fringes were detected at longer baselines also puts an upper limit of $\sim 10 \%$ on the contribution of any point source to the total emission, assuming the disk is fully resolved at these baselines and given that a squared visibility of $1 \%$ can be detected by PIONIER.

Our measurements indicate that the contribution of direct starlight to the total brightness of AR Pup in the $H$ band must be small. The strong brightness increase of AR Pup from the $J$ band to the $H$ band (Figure 1) must thus be dominated by the onset of hot thermal disk emission, rather than the disk becoming optically thin and transmitting direct starlight.

\section{ORCID iDs}

S. Ertel (iD https://orcid.org/0000-0002-2314-7289

D. Kamath (1) https://orcid.org/0000-0001-8299-3402

H. M. J. Boffin (ID https://orcid.org/0000-0002-9486-4840

J. Milli (1) https://orcid.org/0000-0001-9325-2511

G. H.-M. Bertrang (ib https://orcid.org/0000-0001-5127-0172

J. Horner (i) https://orcid.org/0000-0002-1160-7970

J. P. Marshall (iD https://orcid.org/0000-0001-6208-1801

E. Villaver (10 https://orcid.org/0000-0003-4936-9418

S. Xu (i) https:// orcid.org/0000-0002-8808-4282

\section{References}

Alexander, R., Pascucci, I., Andrews, S., Armitage, P., \& Cieza, L. 2014, in Protostars and Planets VI, ed. H. Beuther et al. (Tucson, AZ: Univ. Arizona Press), 475

Almeida, L. A., Jablonski, F., \& Rodrigues, C. V. 2013, ApJ, 766, 11 Anderson, E., \& Francis, C. 2012, AstL, 38, 331

Andrews, S. M., Rosenfeld, K. A., Kraus, A. L., \& Wilner, D. J. 2013, ApJ, 771,129

Ansdell, M., Williams, J. P., Manara, C. F., et al. 2017, AJ, 153, 240

Ansdell, M., Williams, J. P., van der Marel, N., et al. 2016, ApJ, 828, 46

Astropy Collaboration, Robitaille, T. P., Tollerud, E. J., et al. 2013, A\&A, 558, A33

Barenfeld, S. A., Carpenter, J. M., Ricci, L., \& Isella, A. 2016, ApJ, 827, 142
Benisty, M., Stolker, T., Pohl, A., et al. 2017, A\&A, 597, A42 Beuzit, J.-L., Feldt, M., Dohlen, K., et al. 2008, Proc. SPIE, 7014, 701418

Birnstiel, T., Fang, M., \& Johansen, A. 2016, SSRv, 205, 41

Birnstiel, T., Klahr, H., \& Ercolano, B. 2012, A\&A, 539, A148

Bitsch, B., Lambrechts, M., \& Johansen, A. 2015, A\&A, 582, A112

Boffin, H. M. J., Miszalski, B., Rauch, T., et al. 2012, Sci, 338, 773

Bollen, D., Van Winckel, H., \& Kamath, D. 2017, A\&A, 607, A60

Bujarrabal, V., Alcolea, J., Van Winckel, H., Santander-García, M., \& Castro-Carrizo, A. 2013a, A\&A, 557, A104

Bujarrabal, V., Castro-Carrizo, A., Alcolea, J., et al. 2013b, A\&A, 557, L11 Bujarrabal, V., Castro-Carrizo, A., Alcolea, J., et al. 2016, A\&A, 593, A92

Bujarrabal, V., Castro-Carrizo, A., Alcolea, J., et al. 2017, A\&A, 597, L5

Bujarrabal, V., Castro-Carrizo, A., Alcolea, J., \& Van Winckel, H. 2015, A\&A, 575, L7

Bujarrabal, V., Castro-Carrizo, A., Winckel, H. V., et al. 2018, A\&A, 614, A58 Bujarrabal, V., Neri, R., Alcolea, J., \& Kahane, C. 2003, A\&A, 409, 573 Casassus, S., Perez, M. S., Jordán, A., et al. 2012, ApJL, 754, L31 Castelli, F., \& Kurucz, R. L. 2004, arXiv:astro-ph/0405087

Claudi, R. U., Turatto, M., Gratton, R. G., et al. 2008, Proc. SPIE, 7014, $70143 \mathrm{E}$

Cohen, M., Van Winckel, H., Bond, H. E., \& Gull, T. R. 2004, AJ, 127, 2362 Collins, K. A., Kielkopf, J. F., Stassun, K. G., \& Hessman, F. V. 2017, AJ, 153, 77

de Boer, J., Girard, J. H., Canovas, H., et al. 2017, MNRAS, 466, L7

De Marco, O. 2009, PASP, 121, 316

de Ruyter, S., van Winckel, H., Dominik, C., Waters, L. B. F. M., \& Dejonghe, H. 2005, A\&A, 435, 161

de Ruyter, S., van Winckel, H., Maas, T., et al. 2006, A\&A, 448, 641 Debes, J. H., Poteet, C. A., Jang-Condell, H., et al. 2017, ApJ, 835, 205

Deroo, P., Acke, B., Verhoelst, T., et al. 2007, A\&A, 474, L45

Deroo, P., van Winckel, H., Min, M., et al. 2006, A\&A, 450, 181

di Folco, E., Absil, O., Augereau, J.-C., et al. 2007, A\&A, 475, 243

Dohlen, K., Langlois, M., Saisse, M., et al. 2008, Proc. SPIE, 7014, 70143L

Egan, M. P., Price, S. D., Kraemer, K. E., et al. 2003, yCat, 5114, 0

Gaia Collaboration 2018, yCat, 1345, 0

Garaud, P., Meru, F., Galvagni, M., \& Olczak, C. 2013, ApJ, 764, 146

Gesicki, K., Zijlstra, A. A., Szyszka, C., et al. 2010, A\&A, 514, A54

Gezer, I., Van Winckel, H., Bozkurt, Z., et al. 2015, MNRAS, 453, 133

Gielen, C., Bouwman, J., van Winckel, H., et al. 2011, A\&A, 533, A99

Gonzalez, G., Lambert, D. L., \& Giridhar, S. 1997, ApJ, 479, 427

Gorlova, N., Van Winckel, H., Vos, J., et al. 2014, arXiv:1403.2287

Harsono, D., Bjerkeli, P., van der Wiel, M. H. D., et al. 2018, NatAs, 2, 646

Hartmann, L., Calvet, N., Gullbring, E., \& D’Alessio, P. 1998, ApJ, 495, 385 Hauck, B., \& Mermilliod, M. 1998, A\&AS, 129, 431

Helou, G., \& Walker, D. W. (ed.) 1988, Infrared Astronomical Satellite (IRAS) Catalogs and Atlases: The Small Scale Structure Catalog, Vol. 7 (Washington, DC: US Gov. Publ. Office)

Hillen, M., de Vries, B. L., Menu, J., et al. 2015, A\&A, 578, A40 Hillen, M., Kluska, J., Le Bouquin, J.-B., et al. 2016, A\&A, 588, L1 Hillen, M., Menu, J., Van Winckel, H., et al. 2014, A\&A, 568, A12 Hillen, M., Van Winckel, H., Menu, J., et al. 2017, A\&A, 599, A41 Homan, W., Richards, A., Decin, L., et al. 2017, A\&A, 601, A5

Horner, J., Marshall, J. P., Wittenmyer, R. A., \& Tinney, C. G. 2011, MNRAS, 416, L11

Hunter, J. D. 2007, CSE, 9, 90

Jones, E., Oliphant, T., Peterson, P., et al. 2001, SciPy: Open Source Scientific Tools for Python, http://www.scipy.org/

Kamath, D., \& van Winckel, H. 2019, MNRAS, submitted

Kamath, D., Wood, P. R., \& Van Winckel, H. 2014, MNRAS, 439, 2211

Kamath, D., Wood, P. R., \& Van Winckel, H. 2015, MNRAS, 454, 1468

Kamath, D., Wood, P. R., Van Winckel, H., \& Nie, J. D. 2016, A\&A, 586, L5

Kervella, P., Homan, W., Richards, A. M. S., et al. 2016, A\&A, 596, A92

Kervella, P., Montargès, M., Lagadec, E., et al. 2015, A\&A, 578, A77

Kharchenko, N. V., Scholz, R., Piskunov, A. E., Roeser, S., \& Schilbach, E. 2007, yCat, 3254, 0

Kiss, L. L., \& Bódi, A. 2017, A\&A, 608, A99

Kluska, J., Hillen, M., Van Winckel, H., et al. 2018, A\&A, 616, A153

Le Bouquin, J.-B., Berger, J.-P., Lazareff, B., et al. 2011, A\&A, 535, A67

Lee, J. W., Kim, S.-L., Kim, C.-H., et al. 2009, AJ, 137, 3181

Lucy, L. B. 1974, AJ, 79, 745

Manick, R., Van Winckel, H., Kamath, D., Hillen, M., \& Escorza, A. 2017, A\&A, 597, A129

Manick, R., Van Winckel, H., Kamath, D., Sekaran, S., \& Kolenberg, K. 2018, A\&A, 618, A21 
Mermilliod, J.-C., Mermilliod, M., \& Hauck, B. 1997, A\&AS, 124, 349

Miller Bertolami, M. M. 2016, A\&A, 588, A25

Monet, D. G., Levine, S. E., Canzian, B., et al. 2003, AJ, 125, 984

Moshir, M., et al. 1990, BAAS, 22, 1325

Murakami, H., Baba, H., Barthel, P., et al. 2007, PASJ, 59, S369

Mustill, A. J., Marshall, J. P., Villaver, E., et al. 2013, MNRAS, 436, 2515

Nordhaus, J., \& Blackman, E. G. 2006, MNRAS, 370, 2004

Oliphant, T. E. 2006, A Guide to NumPy (USA: Tregol Publishing)

Oomen, G.-M., Van Winckel, H., Pols, O., et al. 2018, A\&A, 620, A85

Osterbart, R., Langer, N., \& Weigelt, G. 1997, A\&A, 325, 609

Pojmanski, G. 2002, AcA, 52, 397

Pollard, K. R., Cottrell, P. L., Kilmartin, P. M., \& Gilmore, A. C. 1996, MNRAS, 279, 949

Price, D. J., Cuello, N., Pinte, C., et al. 2018, MNRAS, 477, 1270

Pulley, D., Faillace, G., Smith, D., Watkins, A., \& von Harrach, S. 2018, A\&A, 611, A48

Richardson, W. H. 1972, J. Opt. Soc. Am., 62, 55

Sahai, R., Morris, M., Sánchez Contreras, C., \& Claussen, M. 2007, AJ, 134,2200

Schulz, B., Marton, G., Valtchanov, I., et al. 2017, arXiv:1706.00448

Scicluna, P., \& Siebenmorgen, R. 2015, A\&A, 584, A108

Sheehan, P. D., \& Eisner, J. A. 2017, ApJL, 840, L12

Skrutskie, M. F., Cutri, R. M., Stiening, R., et al. 2006, AJ, 131, 1163
Soker, N. 2004, in ASP Conf. Ser. 313, Asymmetrical Planetary Nebulae III: Winds, Structure and the Thunderbird, ed. M. Meixner et al. (San Francisco, CA: ASP), 562

Soker, N. 2006, PASP, 118, 260

Stolker, T., Dominik, C., Avenhaus, H., et al. 2016, A\&A, 595, A113

Thalmann, C., Schmid, H. M., Boccaletti, A., et al. 2008, Proc. SPIE, 7014, $70143 \mathrm{~F}$

Turner, N. J., Fromang, S., Gammie, C., et al. 2014, in Protostars and Planets VI, ed. H. Beuther et al. (Tucson, AZ: Univ. Arizona Press), 411

van Leeuwen, F. 2007, A\&A, 474, 653

van Lieshout, R., Kral, Q., Charnoz, S., Wyatt, M. C., \& Shannon, A. 2018, MNRAS, 480, 2784

van Winckel, H. 2003, ARA\&A, 41, 391

van Winckel, H., Lloyd Evans, T., Briquet, M., et al. 2009, A\&A, 505, 1221 Waelkens, C., Lamers, H. J. G. L. M., Waters, L. B. F. M., et al. 1991, A\&A, 242,433

Waelkens, C., Van Winckel, H., Waters, L. B. F. M., \& Bakker, E. J. 1996, A\&A, 314, L17

Wang, Z., Chakrabarty, D., \& Kaplan, D. L. 2006, Natur, 440, 772

Wittenmyer, R. A., Horner, J., \& Marshall, J. P. 2013, MNRAS, 431, 2150

Wolszczan, A., \& Frail, D. A. 1992, Natur, 355, 145

Zuckerman, B., Melis, C., Song, I., et al. 2008, ApJ, 683, 1085

Zuckerman, B., Rhee, J. H., Song, I., \& Bessell, M. S. 2011, ApJ, 732, 61 